\title{
REDISTRIBUCIÓN DE TIERRAS EN SAN JOSÉ DE COLLANA PAUCARCOLLA PUNO PERÚ
}

\author{
REDISTRIBUTION OF LANDS IN SAN JOSÉ DE COLLANA PAUCARCOLLA PUNO PERU
}

\author{
Fermin Francisco Chaiña Chura
}

\begin{abstract}
RESUMEN
La presente investigación tuvo como propósito, analizar y comprender el proceso de parcelación de áreas adjudicadas de la ex Sociedad Agrícola de Interés Social Vilque a favor de la comunidad en estudio, partiendo de la aplicación de la Ley ${ }^{\circ} 17716$ de Reforma Agraria y en el marco del Decreto Legislativo $\mathrm{N}^{\circ}$ 002, Ley de Promoción y Desarrollo Agrario, después de diez años de iniciado el proceso de cambio de la estructura agraria en Perú. Se utilizó el procedimiento metodológico de análisis-síntesis, a fin de descomponer las fases por los que discurrió este proceso de redistribución: adjudicación, tenencia y parcelación. Principales resultados: despojo de tierras de comunidades campesinas por parte del sistema de hacienda, que generó conflictos sociales en Perú y al convertirse en crisis política creó condiciones favorables para el establecimiento de un gobierno militar, que promulgó la Ley Agraria y en otra gestión, la de Promoción y Desarrollo Agrario, que apertura la reestructuración y la parcelación de tierras; proceso que se realizó a través de una Comisión de Parcelación, que preparó y entregó parcelas vía sorteo a 74 socios, en un aproximado de ocho hectáreas a cada uno; en parcelas de pampa, ladera y cerro. Los factores que influenciaron en este proceso fueron las condiciones políticas favorables y la autonomía de la Asamblea General de la Comunidad.
\end{abstract}

Palabras clave: Adjudicación, agraria, parcelación, reestructuración, reforma.

\section{ABSTRACT}

The present investigation, Redistribution of land in San Jose de Collana Paucarcolla Puno-Peru, had as its purpose, to analyze and understand the process of subdivision of areas adjudicated of the former Agricultural Society of Social Interest Vilque in favor of the community under study, starting from the application of Law N017716 of Agrarian Reform and within the framework of Legislative Decree N0002, Law of Promotion and Agrarian Development, after ten years of the process of change of the agrarian structure in Peru. The methodological analysis-synthesis procedure was used, in order to decompose the phases through which this redistribution process took place: adjudication, tenure and subdivision. Main results: It is the dispossession of lands of peasant communities by the hacienda system, which generates social conflicts in Peru and, by becoming a political crisis, creates favorable conditions for the establishment of a military government, which promulgates the Agrarian Law and in another management the one of Promotion and Agrarian Development, that open the restructuring and the parcelling of lands; process that is carried out through a Parceling Commission, which prepares and delivers parcels through lottery to a total of 74 partners, an approximate of eight hectares each; in pampa, hillside and hill plots. The factors that influenced this process were the favorable political conditions and the autonomy of the General Assembly of the community.

Keywords: Adjudication, agrarian, subdivision, restructuring, reform.

\section{INTRODUCCIÓN}

El desarrollo global de la sociedad ha producido profundos cambios sociales, científicos y tecnológicos. Este proceso ha tenido efectos en la estructura agraria del Perú y en el marco de tal situación en el sistema del mundo contemporáneo. El tema de investigación es la redistribución de tierras en la Comunidad Campesina de San José de Collana Paucarcolla, Puno-Perú, con el objetivo de analizar y comprender el proceso de parcelación de áreas adjudicadas, luego de la desintegración de la Sociedad Agrícola de Interés Social (SAIS) Vilque, a

${ }^{1}$ Doctor en Educación, Magister Scientiae en Desarrollo Rural. Facultad de Ciencias Sociales, Universidad Nacional del Altiplano, Puno-Perú. E-mail.: fchaina1@hotmail.com

Presentado: 23/11/16 Aprobado: 25/08/17 
favor de la organización comunal, adjudicación realizada por el Ministerio de Agricultura a través de la Dirección General de Reforma Agraria y Asentamiento Rural (DGRAIAR), después de un periodo de tiempo, los beneficiarios deciden parcelar dicho fundo entre sus socios en el marco de las disposiciones legales vigentes y al amparo de la autonomía de la Asamblea General de Socios de la Comunidad.

No se ha identificado estudios sobre parcelación de tierras de reforma agraria en áreas comunales, lo que resulta una limitante. Esta investigación se justifica porque genera conocimiento de los procesos sociales de parcelación de tierras desarrollados en el altiplano de Puno, cuyos resultados servirán para actividades académicas, el diseño de acciones de intervención y constituirán la base de futuras investigaciones en el área.

\section{MATERIALES Y MÉTODOS}

Este tipo de investigación por su naturaleza es de nivel descriptivo-explicativo, porque se pretende conocer la dimensión social del proceso de redistribución de tierras en el ámbito de estudio. Se identifica el predio que fue adjudicado a la comunidad, como resultado de la reestructuración agraria por el Ministerio de Agricultura a través de la DGRAIAR, instancia encargada de la tramitación de solicitudes para el cambio de modelo, proceso que discurre por las siguientes fases:

a) LaAsamblea General de Socios en sesión extraordinaria convocada, para tal efecto, aprueba la intención de cambio de modelo empresarial con la firma en acta de dos tercios de socios hábiles.

b) Para el proceso de cambio de modelo empresarial se requiere la siguiente documentación:

- Plano de conjunto a escala 1/25,000, con el detalle del área total de los predios y el número de parcelas debidamente demarcadas y numeradas.

- Estatutos de la nueva empresa

- Acta de Asamblea General de intención de cambio firmado por los dos tercios de los socios hábiles, con legalización notarial.

- Datos sobre la titulación, el reconocimiento y el número de socios hábiles.

- Inventario actualizado valorizado antes de la aprobación del cambio de modelo.

- Estado económico financiero, con el último balancegeneral.

En esta investigación, se utilizó los planos de parcela- ción de tierras; la colecta de información necesaria se desarrolló en base a la revisión y análisis del libro de actas de la comunidad; el procesamiento de la información se efectuó en base a datos resultantes de la observación en el ámbito de estudio. Cuando se nombra comunidad o San José de Collana, en ambos casos se refiere a la Comunidad Campesina de San José de Collana.

\section{RESULTADOS Y DISCUSIÓN}

\section{Proceso de reforma agraria peruana}

Es el clamor por la tierra de parte de campesinos de los países de América Latina y El Caribe, lo que preocupa a sus respectivos gobiernos, debido a que entre 1955 y 1965 se suscitan movimientos campesinos y conflictos sociales que tienden a crecer.

En Perú hasta la década de 1960, se produce una extrema concentración de la propiedad agraria en el sistema de haciendas, resultante del despojo de tierras, principalmente en el altiplano de Puno, por un lado y por otro, el constante fraccionamiento de tierras en las comunidades campesinas, por crecimiento demográfico. Estos factores dan origen a los problemas sociales en esta década, proceso de organización y conflictos rurales en la sierra central, en los valles de la Convención y Lares, y en el resto de Perú; al convertirse en crisis política se crean condiciones favorables para la intervención de un gobierno militar.

Entonces, es el General Juan Velazco Alvarado, quién da el golpe de estado, estableciendo un gobierno militar el 03 de octubre de 1968 para asumir la tarea de pacificación del medio rural con la aplicación de un conjunto de medidas estratégicas, donde uno de los componentes es la Reforma Agraria a través de la promulgación de la Ley $\mathrm{N}^{0} 17716$, dada el 24 de junio de 1969.

\section{Reestructuración agraria}

Los procesos de reestructuración agraria en Puno, se implementa en el marco del Decreto Legislativo $\mathrm{N}^{\circ} 002$ Ley de Promoción y Desarrollo Agrario del 25 de noviembre de 1980. Esta norma especifica en los artículos 78 y 80 sobre la parcelación, el cambio de modelo en las empresas campesinas asociadas y faculta a la DGRAIAR la responsabilidad de normar la implementación de estos procesos de parcelación, en el marco del art. 80 que precisa: "las empresas asociativas podrán decidir libremente sobre el modelo empresarial que mejor convenga al interés de sus asociados". En el marco de esta norma, se fraccionó la SAIS Vilque entre sus socios constituidos por ex-feudatarios y comunida- 
des campesinas. Esta empresa agraria, cuya jurisdicción abarcó parte de los distritos de Vilque, Tiquillaca y Paucarcolla, adjudicó tierras a través del Ministerio de Agricultura a sus comunidades socias como es el caso de San José de Collana, que recibió un total de 495 hectáreas con $7,743.20 \mathrm{~m}^{2}$ parte del fundo Chincheros.

Estas tierras se trabajaron como empresa comunal por un periodo aproximado de diez años en la producción agrícola y la crianza de vacunos, cuya producción de leche incrementó los ingresos familiares de los socios comuneros; posteriormente, en la Comunidad de San José de Collana se decide en Asamblea General de Socios realizar el proceso de parcelación.

En consecuencia, las condiciones políticas que influenciaron los procesos de reestructuración agraria y la posterior parcelación del área comunal, fueron: la Constitución Política de Perú de 1993, que elimina la inalienabilidad de las tierras comunales; Decreto Legislativo $\mathrm{N}^{\circ} 002$ Ley de Promoción y Desarrollo Agrario, mediante el art. 80 apertura los procesos de reestructuración del agro; Decreto Supremo $\mathrm{N}^{\circ}$ 001-82-AG del 04 de enero de 1982 Reglamento de la Ley de Promoción y Desarrollo Agrario, que detalla el proceso de reestructuración agraria; Decreto Legislativo $\mathrm{N}^{\circ} 653$ Ley de Promoción de la inversión en el sector agrario del 01 de agosto de 1991, el mismo que regula la formalización de los predios con extensiones mayores y que fueron adquiridos a título oneroso; Ley $\mathrm{N}^{\circ} 24657$ de deslinde y titulación; Ley General N ${ }^{0} 24656$ de Comunidades Campesinas; asimismo, la autonomía de la Asamblea General de Socios de la Comunidad, crean condiciones políticas favorables para procesos de parcelación de tierras.

\section{Proceso de preparcela}

Este proceso se desarrolló entre los años 1980 y 1996, antes del proceso de parcelación, que consistió en una modalidad de trabajo en tierras del ámbito comunal distribuidas en calidad de pre parcela, con carácter transitorio y vía, la siguiente ruta: 1) se preparó parcelas de tamaño determinados para cada uno de los 74 socios; 2) la pre parcela tenía una particularidad de estar destinado sólo a actividades agrícolas, únicamente para cultivos y no se entregó espacios de pastos naturales para la ganadería familiar, debido a que los pastizales se destinaron para el ganado de la comunidad, maneja- da por el sistema de turnos por cada uno de los socios; 3) tuvo una duración muy temporal de aproximadamente diez años, estos espacios de pre parcela fueron trabajados desde la limpieza del suelo, como una tarea de habilitación para cultivos futuros, orientado a la parcelación definitiva y 4) en la parcelación, estas áreas no siempre correspondieron a los mismos socios que venían trabajando en proceso de pre parcela.

\section{Proceso de parcelación}

La parcelación de tierras en San José de Collana transitó por el siguiente proceso: nominación de la Comisión de Parcelación, fijación de áreas a parcelar, preparación de lotes, sorteo de parcelas por grupos de socios y formalización de la parcelación.

a) Parcelación de tierras correspondió al área adjudicada a la Comunidad San José de Collana por el Ministerio de Agricultura a través de la DGRAIAR, en un total de 495 hectáreas con $7,743.20 \mathrm{~m}^{2}$. El espacio comprendió superficies de pampa, ladera y cerro; donde se preparó 10 zonas para parcelar entre 74 socios en cada espacio.

b) Proceso de parcelación se desarrolló en el marco de la Asamblea General de Socios, que se inició en dos zonas: siguiendo la ruta de este a oeste, de la zona de pampa hacia la zona alta. Primeramente se parceló la zona $\mathrm{N}^{0} 1$, ubicada en el lado este de la Carretera Panamericana Sur Puno-Juliaca denominada "Kollpachupa", continuando con la zona $\mathrm{N}^{0} 2$ denominado "Rielpata" por su cercanía a la línea férrea, tramo Puno-Juliaca. Este proceso inicial de redistribución de tierras en dos espacios se desarrolló por sorteo entre los 74 socios, experiencia de sorteo general que no agradó al grupo directivo, debido a que la suerte no acompañó a sus aspiraciones de tener la mejor parcela 0 el mejor lote con buena calidad de pastos naturales y una ubicación aparente para cultivos, puesto que la Comisión de Parcelación tenía que satisfacer esas pretensiones, siendo necesario cambiar de estrategia; por lo que, se trasladó a la zona alta de "Titiake" y en otra fecha, avanzando con el proceso de parcelación en dirección contraria a la ruta inicial. Esta vez se avanzó de oeste a este, de la zona alta hacia la zona pampa, aplicando la modalidad de sorteoporgrupos. 


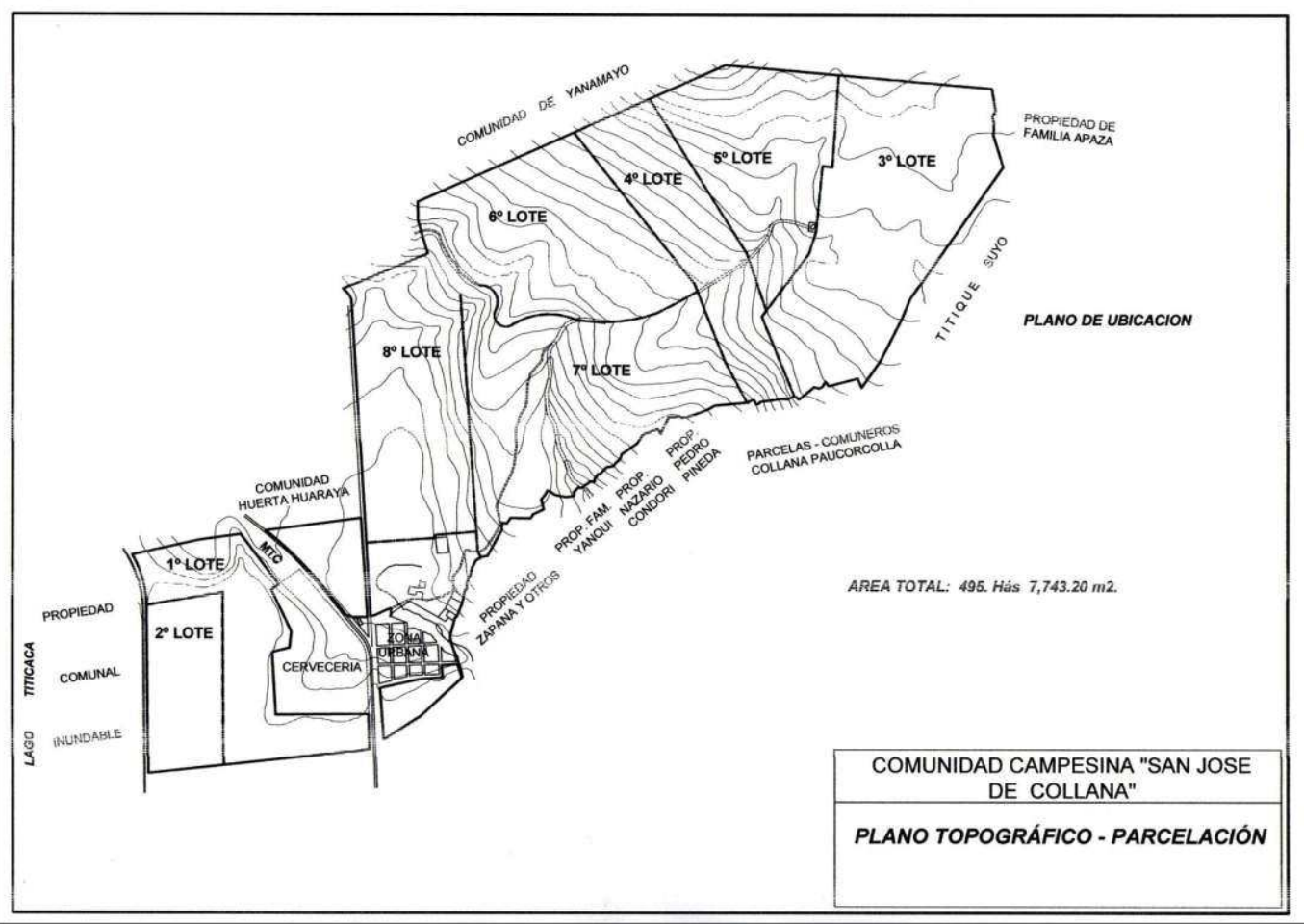

Figura 1. Zonificación de espacios de pampa, ladera y cerro

Fuente: Elaborado en base a copia de plano entregado a cada socio

En este proceso, cada zona o lote se dividió en 74 parcelas, para entregar uno por socio, tal como se aprecia en la figura 1; entonces cada socio recibió una parcela en cada zona, tal como se observa en la figura 2, que corresponde a la zona 8 de "Huertopampa", donde se visualiza como ejemplo las 74 parcelas.

El grupo A resultó ubicado en los mejores lotes individuales y el resto de socios en parcelas de tierra de aparente menor calidad, con pastos naturales y la accesibilidad entre otros factores.

Estas prácticas de parcelación, no solo se produce en San José de Collana, sino en el ámbito provincial; al respecto, se tiene evidenciado estos estilos de distribución de tierras; por ejemplo, en las pampas de Illpa en la Comunidad de San Juan de Huata en la parcelación de tierras de la ex hacienda Moro y otros, lo que fue juris- dicción de la SAIS Buenavista. Otro ejemplo, es la parcelación de la ex hacienda Collacachi, lo que fue la SAIS Puno.

c) Formalización de las parcelas adjudicadas en las dos modalidades de sorteo, que bordea las ocho hectáreas. Los 74 socios tienen ahora un instrumento legal, que es la Escritura Pública de la Notaría Centeno Zavala, en la que están descritas y formalizadas las características de cada una de las parcelas entregadas en un solo instrumento público.

d) Culminado el proceso de parcelación del área comunal que correspondió a la comunidad San José de Collana, cada socio es beneficiario con un aproximado de 10 a 13 lotes o parcelas, según fue el caso; ubicados en las siguientes zonas: 
Tabla 1. Área de la parcela entregada a cada socio por zona

\begin{tabular}{llrl}
\hline $\begin{array}{l}\mathbf{N}^{\mathbf{0}} \\
\text { Ord. } \\
\text { Zona }\end{array}$ & Nombre de la & $\begin{array}{c}\text { Área de } \\
\text { cada lote } \\
\left(\mathbf{m}^{\mathbf{2}}\right)\end{array}$ & Zona \\
\hline 1 & Kollpachupa & $4,056.10$ & pampa \\
2 & Rielpata & $5,513.30$ & pampa \\
3 & Titiakepata & $10,280.00$ & cerro \\
4 & Saihuani & $6,780.00$ & cerro \\
5 & Tarucani & $6,626.00$ & cerro \\
6 & Ichupampa & $8,747.00$ & cerro \\
7 & Sancayuni & $11,250.00$ & cerro \\
8 & Huerto pampa & $8,000.00$ & pampa ladera \\
9 & Quinsa pujio parte cerro & $1,369.80$ & ladera \\
10 & Quinsa pujio parte pampa & $1,164.60$ & pampa \\
11 & Zona urbana Villa San José & 600.00 & ladera \\
12 & Z.u.: Inmaculada Concepción & $1,040.00$ & ladera \\
13 & Lotes compensados a 12 socios & 200 y150 & pampa waru waru \\
\hline
\end{tabular}

Fuente: Elaborado en base al acta del 22 de marzo de 1998

e) Las parcelas adjudicadas fueron gestionadas por la unidad familiar y conducidas como componentes de estas economías rurales. Los socios de San José de Collana tienen ahora parcelas en diferentes ámbitos, tanto en la comunidad y fuera de ella, en la parcialidad de Collana que es un ámbito extra comunal, jurisdicción del distrito de Paucarcolla. Las tierras de los socios fueron distribuidas y ubicadas en zonas agroecológicas circundantes al Lago Titicaca 0 zona pampa, así como en las zonas de ladera y cerro; constituida por parcelas de cultivos, parcelas de pastos naturales y pastos cultivados para la ganadería familiar, entre otros.

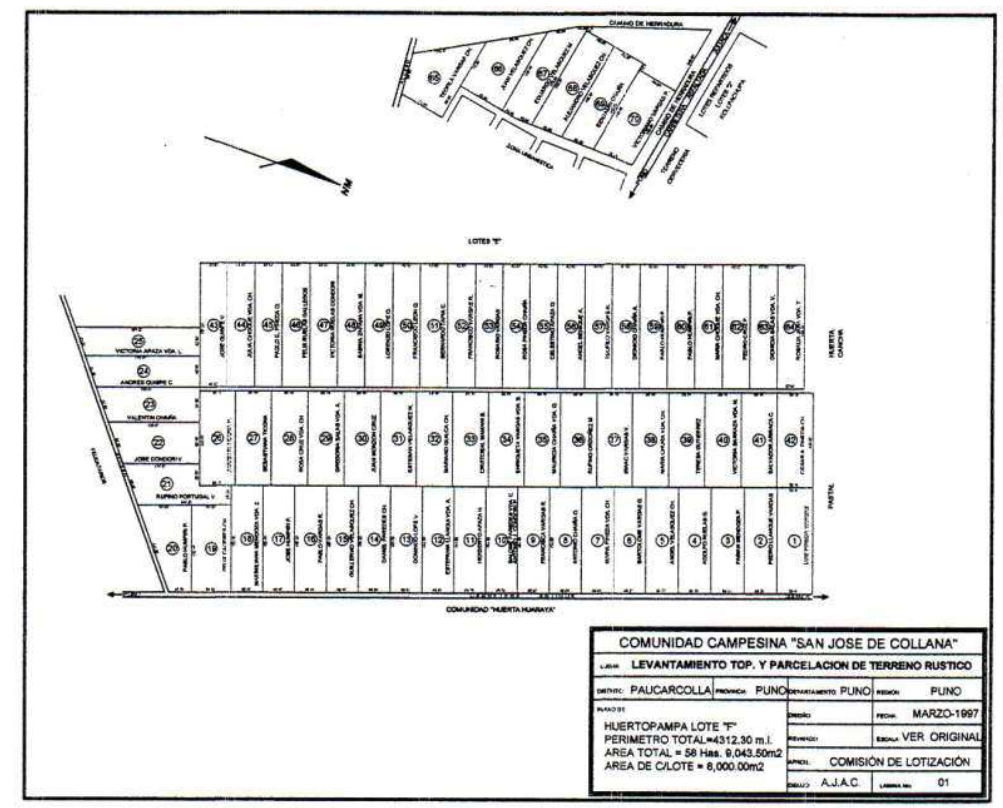

Figura 2. Parcelación de la zona Huertopampa*

Fuente: Elaborado en base a copia de plano entregado a cada socio.

*: En la zona 8 de "Huertopampa", cada lote tiene $8,000 \mathrm{~m}^{2}$, que fue entregado a cada socio; la sumatoria de las trece parcelas constituyen el área total en un aproximado de ocho hectáreas, que recibió cada uno de los 74 socios. 


\section{DISCUSIÓN}

\section{Proceso de reforma agraria peruana}

En los países de América Latina, los procesos de reforma agraria se constituyen como el resultado de la reacción de los comuneros y parceleros frente al abuso de parte de los hacendados, que les despojaron sus mejores tierras. En Perú, se tiene evidencia de estos procesos, como el caso del fundo Chincheros del distrito de Paucarcolla en Puno, donde la propiedad de la familia Vargas ancestralmente tenía sus áreas de pastoreo; en la actualidad, son espacios que maneja el sistema de hacienda como propiedad privada (testimonio del señor Pedro Llanqui Vargas). Son estos despojos y otros similares, lo que generó movimientos campesinos que preocupó a sus respectivos gobiernos, debido a que se suscitan conflictos sociales que tienden a crecer. Los problemas sociales de los valles de la Convención y Lares, entre otros movimientos al convertirse en crisis política, crearon condiciones favorables para la intervención del gobierno militar.

Al respecto, Nercesian (2017) considera que para enfrentar esta situación y asumir la tarea de pacificación del medio rural, las Fuerzas Armadas ocuparon el Palacio de Gobierno la madrugada del 3 de octubre de 1968 y destituyeron al desprestigiado gobierno de Belaúnde Terry (19631968) de la mano del militar nacionalista Juan Velasco Alvarado (1968-1975). América Latina vivía momentos convulsionados. Hacía poco menos de diez años que se había desencadenado la Revolución Cubana y en la región se vivía un clima de efervescencia política y social.

El General Juan Velazco Alvarado, después del Golpe de Estado, establece un gobierno militar, aplica un conjunto de medidas estratégicas, donde uno de los componentes es la Reforma Agraria a través de la Ley N ${ }^{\circ} 17716$ promulgada el 24 de junio de 1969 con el principio de: "Campesino, el patrón ya no comerá más de tu pobreza".

Esta Ley de Reforma Agraria está basada en las experiencias de los gobiernos anteriores. En referencia a este punto, es posible leer partes del primer artículo sobre la forma integral como es planteado dicho proceso: Artículo $1^{\circ}$.- "La Reforma Agraria es un proceso integral y un instrumento de transformación de la estructura agraria del país, destinado a sustituir los regímenes de latifundio y minifundio por un sistema justo de propiedad, tenencia y explotación de la tierra, que contribuya al desarrollo social y económico de la nación, mediante el ordenamiento agrario que garantice la justicia social en el campo y aumente la producción y la productividad del sector agropecuario, elevando y asegurando los ingresos de los campesinos para que la tierra constituya para el hombre que la trabaja, base de su estabilidad económica, fundamento de su bienestary garantía de su dignidad y libertad". Esta Ley de reforma agraria reconoce el derecho de propiedad privada de la tierra a través de pago a los hacendados vía deuda agraria, manteniendo a los campesinos al margen del control del Estado (Valderrama, 1978).

En el proceso de aplicación de esta ley, entre junio de 1969 y junio de 1979 se expropiaron 15,826 fundos y más de 9 millones de hectáreas (9'688,763 ha). La mayor parte de esta área se adjudicó a 370 mil beneficiarios (Eguren, 1980). Se constituyeron Empresas de Reforma Agraria, como las Sociedades Agrícolas de Interés Social (SAIS), las Cooperativas Agrarias de Producción (CAP) y Empresas Rurales de Propiedad Social (ERPS).

Al respecto, Matos (1980) precisa que en 1979, a diez años de iniciado el programa de Reforma Agraria, este se encuentra prácticamente paralizado. Situación acorde con la orientación del gobierno militar que, al revocar la política del periodo del general Velasco ha eliminado la mayoría de las reformas, quedando la agraria como la única hasta ahora no sustantivamente revertida.

El autor refiere, que a partir de 1975, a causa del giro que significa el agotamiento del proyecto militar sobre el cambio de la sociedad peruana, que conduce al abandono del original programa agrario; provoca en 1980 nuevos movimientos de organizaciones campesinas como el caso de los movimientos de la provincia de Melgar en la Región Puno, y la nueva crisis económica y política general a nivel nacional, que evidencia la incapacidad de las reformas para resolver los problemas planteados; se esbozan entonces, nuevas alternativas de política agraria, lo que conlleva al tema de reestructuración agraria.

\section{Proceso de parcelación, dominio y poder}

El proceso de redistribución de tierras o parcelación se inició nominándose una Comisión de Parcelación; ente que prepara los respectivos planos de parcelas por zonas o lotes, cada una de estas áreas contiene 74 parcelas para la misma cantidad de socios. En pleno proceso de sorteo general, surgió la modalidad de sorteo por grupos de socios comuneros en función al trabajo y participación en procesos de gestión para conseguir las tierras de Reforma Agraria, lo que es aceptado por la mayoría; se procedió al sorteo y entrega de parcelas a cada uno de los socios por grupos.

Cuando se menciona grupos, el grupo A "dirigencial" está constituido por gestores, ex presidentes y autoridades; el grupo B integrado por socios "sin faltas", grupo C por socios "con faltas". ¿Por qué los grupos?, ¿Es de 
interés de los gestores? o ¿Es expresión de dominio del grupo dirigente? En este caso, es la expresión del interés del grupo dominante; el establecimiento de grupos es la justificación ante el fracaso del primer día de sorteo, donde al segmento de poder, obtuvieron parcelas de "baja calidad" en pastos naturales y para la agricultura por ser áreas rocosas sin aptitud agropecuaria; situación que los obligó a cambiar de estrategia. En otra fecha (segundo día de sorteo), aquí previo al sorteo, en el interior de cada zona o lote que contiene 74 parcelas, se seleccionó la cantidad de parcelas requeridas para cada grupo, las de mejor calidad para el grupo A, las de calidad regular para el grupo B y las de baja calidad para el grupo C; estrategia que sí resultó apropiado y funcional para las aspiraciones del grupo dominante. El sorteo del primer día quedó totalmente válido, el grupo de dominio conserva sus parcelas de "baja calidad" hasta hoy; pero, el resto de sus parcelas resultantes del sorteo por grupos son de "buena calidad". Cada uno de los socios recibió un área total de ocho hectáreas aproximadamente.

Entonces, queda demostrado que la dominación es la resultante de la capacidad de imponer algo, es un proceso persuasivo que utiliza hasta procedimientos psicológicos, todo por ejercer control y dominio; si una persona o grupo acepta es la evidencia de dominio. En términos de Quijano (2000) es imposible la democratización real.

\section{Gestión del espacio parcelado}

Desde entonces hasta hoy, los socios de la comunidad vienen trabajando sus parcelas en diferentes zonas principalmente en actividades agropecuarias. Se indica, que en el caso de San José de Collana, las parcelas han contribuido al bienestar social de las unidades familiares, en estas parcelas se desarrollan trabajos de agricultura y crianza de la ganadería familiar. Los socios de esta comunidad viven en el área urbana, que tiende a convertirse en Centro Poblado en el lugar denominado "Patallani", donde se establece la "Villa San José", desde este núcleo realizan sus actividades cotidianas, lo que constituye la nueva ubicación de su habitad al margen de su vivienda de la parcialidad de Collana, que es ámbito extra-comunal, casi abandonado por las escasas condiciones para el desarrollo de sus actividades agropecuarias, convirtiéndose la Villa San José con mayores ventajas por su accesibilidad hacia los centros urbanos de Puno y Juliaca principalmente; ubicación aparente que permite el funcionamiento de estos sistemas agroeconómico familiares en el interior de comunidades campesinas (Chaquilla, Toninay Chaguares, 1982).

Al respecto, Figueroa (2010) refiriéndose a la calidad del suelo y la fragilidad de los ecosistemas andinos, considera lo siguiente: "La mayoría de la población que habita actualmente en la sierra peruana obtiene su subsistencia de una agricultura que lleva a cabo en tierras marginales, en ladera sin agua, en suelos pelados. Solo la economía campesina es viable en estas tierras". Efectivamente, estos suelos en épocas pasadas eran fértiles, porque se practicaba el uso racional en estos espacios; hoy tiende a convertirse en suelos empobrecidos, debido al uso agrícola bajo lógicas contemporáneas.

\section{Perspectivas y tendencias}

Hasta la década de 1980, la comunidad fue la principal forma de organización rural (Hall, 2013), hoy esta modalidad de organización tiende a ser conjugada con sistemas de producción familiar. En este contexto, las tendencias futuras de esta comunidad están consideradas en su plan estratégico, en cuya misión se precisa lo siguiente: "Somos una comunidad organizada de los mejores agricultores y ganaderos, buscamos el desarrollo integral a través del procesamiento de nuestros productos, la promoción de la artesanía y el turismo rural; haciendo empresa para mejorar los ingresos y contribuir al progreso de la Villa San José, la región y del país; cultivando el respeto, honradez y laboriosidad".

Esta misión será viable, cuando se implemente en el marco de una concepción de desarrollo humano sostenible a nivel local, donde los actores estratégicos sean la persona, la comunidad y el sector institucional, considerando que el desarrollo de la comunidad es un instrumento, que mediante la organización y la educación de las colectividades promueve, entre otras cosas, la participación consciente de la población en el planeamiento y ejecución de programas de beneficio colectivo (Ander-Egg, 2003).

Por otro lado, Gonzales (2011) precisa que lo más importante es, qué se entiende por desarrollo, que a menudo se confunde por incremento del producto o del ingreso por persona, definición que deja de lado las desigualdades y reduce el problema de desarrollo a los bajos ingresos; es decir, a la pobreza. El verdadero desarrollo es aquel que mejora las capacidades de las personas, les permite vivir en libertad, este es el desarrollo humano. En los últimos diez años, las comunidades campesinas pasaron a ser sujeto de un nuevo contexto: el proceso de expansión de las industrias extractivas en territorios comunales (Burneo, 2012).

Al respecto, Leff (2010) complementa lo siguiente, en los tiempos actuales los conflictos sociales se originan en defensa del medio ambiente provocado por el extractivismo que lo contamina; los pueblos se movilizan en contra de la contaminación ambiental, en defensa del suelo, del agua, de la vida. 
En síntesis, hasta la década de 1980 la lucha era por la tierra; hoy es por la defensa del medio ambiente, del agua y de la vida. Por tanto, el verdadero desarrollo de las comunidades rurales sigue siendo una tarea pendiente y compleja.

\section{CONCLUSIONES}

La concentración de tierras en el sistema de haciendas y el constante fraccionamiento en comunidades campesinas, fueron factores que originaron los problemas sociales en Perú durante la década de 1960; al convertirse en crisis política crearon condiciones para la intervención de un gobierno militar, que promulga la Ley Reforma Agraria $\mathrm{N}^{\circ} 17716$, proceso que implementó la afectación del sistema de haciendas, adjudicación a empresas agrarias como las SAIS y otras organizaciones agrarias; luego la crisis económica, la crisis política y los conflictos rurales de la década de 1980 dan inicio a la reestructuración agraria.

El proceso de redistribución se realizó con la nominación de una Comisión de Parcelación, que preparó los respectivos planos de parcelas por zonas y los grupos de socios comuneros beneficiarios en función al trabajo 0 jornadas acumuladas y participación en gestión. Fase que se concretizó, vía sorteo general de parcelas en un primer momento y sorteo por grupos en la segunda etapa, en ambos casos entre los 74 socios comuneros en parcelas de las zonas pampa, ladera y cerro; cada comunero recibe un promedio de ocho hectáreas; proceso que culmina con la formalización en Escritura Pública Notarial; falta culminar el proceso con la inscripción en SUNARP.

Los socios de la Comunidad San José de Collana, actualmente vienen trabajando sus parcelas en diferentes zonas, desarrollando la agricultura y la ganadería familiar. Estas tierras resultantes del proceso de parcelación han contribuido a la satisfacción de las necesidades básicas de la unidad familiar. Los factores que influenciaron en los procesos de reestructuración y parcelación de tierras del área comunal, fueron las condiciones políticas favorables expresadas en la normatividad por un lado y por otro, la autonomía de la Asamblea General de Socios de la Comunidad.

\section{REFERENCIAS BIBLIOGRÁFICAS}

Ander-Egg, E. (2003). Metodología y práctica del desarrollo de la comunidad. ¿Qué es desarrollo de la comunidad?. Buenos Aires: LUMEN Hvmanitas.

Burneo, M. (2012). Elementos para volver a pensar lo comunal: nuevas formas de acceso a la tierra y presión sobre el recurso en las comunidades campesinas de Colán y Catacaos. ANTHROPOLOGICA/AÑO XXXI, $\mathrm{N}^{\circ} 31$ : 15-41.

Chaquilla, 0., Tonina, T., Chahuares, E. (1982). Modelo del sistema agroeconómico familia-predio en comunidades campesinas del Altiplano Peruano. Seminario sobre planificación Regional de la Investigación Agropecuaria. Puno.

Eguren, F. (1980). Reforma agraria y desarrollo rural en el Perú. Lima: Instituto de Estudios Peruanos.

Figueroa, A. (2010). Limites del desarrollo agrario de la sierra peruana. Rev. Economía Vol. XXXIII, $\mathrm{N}^{\circ}$ 66: 157-160.

Gonzalez, E. (2011). Qué entendemos por desarrollo económico o de desarrollo [mensaje en un blog]. Recuperado de http://blog.pucp.edu.pe/blog/ desarrollohumano/2011/06.

Hall, I. (2013) La reforma agraria, entre memoria y olvido (Andes sur peruanos). ANTROPOLOGICA/ AÑ̃O XXXI, $\mathrm{N}^{0} .31: 101-125$.

Leff, E. (1998). Ecología y capital. Racionalidad ambiental, democracia participativa y desarrollo sustentable. Madrid y México: UNAM.

Maleta, H. (2009). Epistemología aplicada: Metodología y técnica de producción científica. Lima Perú: CIES. CEPES. Universidad Pacífico.

Matos, J. (1980). La reforma agraria en el Perú. Lima Perú: IEP.

Mayer, E. (2009). Cuentos feos de la reforma agraria peruana. Lima: IEP: CEPES.

Molestina, C. (1988). Fundamentos de comunicación científica y redacción técnica. San José, Costa Rica: IICA.

Nercesian, I. (2017). La experiencia de Velasco Alvarado en Perú (1968-1975): intelectuales y política. Una aproximación. Rev. E-1@tina,Vol. 15, № 59, Buenos Aires.

Quijano, A. (2000). El fantasma del desarrollo en América Latina. Rev. Venez. de Econ. y Ciencias Sociales, Vol. 6 $\mathrm{N}^{0} 2: 73-90$.

Serna, J. y otros. (2001). Gestión en Programas Sociales. Lima Perú: PROMUDEH.

Valderrama, M. (1978). Movimiento campesino y reforma agraria en el Perú. Nueva Sociedad Nro.35: 103-113. 